\title{
On the Translation Strategies of Movie Dubbing and Subtitling: A Frequency Analysison Explicitation in Translation
}

\author{
Marzieh Bagheri ${ }^{1, *}$, Azadeh Nemati ${ }^{2}$ \\ ${ }^{1}$ Department of Translation Studies, Science and Research, Branch, Islamic Azad University, Fars, Iran \\ ${ }^{2}$ Department of English Language Teaching, Jahrom Branch, Islamic Azad University, Jahrom, Iran \\ *Corresponding Author: marziehbagheri58@yahoo.com
}

Copyright @ 2014 Horizon Research Publishing All rights reserved.

\begin{abstract}
Dubbing, also known as voice-overs, is the most common way of presenting the audience with the materials in a movie. As this process requires time, budget, and a team of translators, voice-overs, voice recorders, etc., producers sometimes tend to release the movies in other languages through subtitling. This is a rather easier procedure, presenting the audience with the written translated text of the material being spoken and happening in a movie. Several movies are then being processed through these common procedures in different countries. However, the question still remains as to what strategies make up the most important issues in movie translation. Explicitation vs. implicitation, domestication vs. foreignization, etc., are just some of these strategies. In this regard, many models have been presented by translation theorists (e.g., Klaudy 2008, Venuti 20004, Larson 1998, etc.). In line, the present study tended to investigate the explicitation strategies pointed out by Klaudy (2008) while dealing with the act of dubbing on the one hand, and subtitling on the other. In this regard, "Harry Potter and the Philosopher's Stone" was selected as the corpus of the study. Using Klaudy's (ibid.) model of translation explicitation, the cases were detected and further placed within the corresponding categories. Having a frequency analysis on the collected data, the results revealed statistically significant differences among the frequencies of the strategies in the dubbed and subtitled versions of the movie. The results also revealed that 'obligatory explicitation' was the most frequent strategy used in both versions by Iranian translators.
\end{abstract}

Keywords Translation Strategies, Translation Process, Explicitation, Frequency Analysis, Harry Potter

\section{Introduction}

With the growing pace of technology, a great deal of information is being presented to the audience each and every day. One of the most common ways to such distribution is through audio-visual (AV) means. These mostly include TV, cinema and the internet. Although the trend is changing with the rise of technology, dubbing and subtitling are still two of the most usual ways of presenting a movie to the audience in different countries of the world. Subtitling is merely known as providing the written translation of the script under the screen. On the dubbing side, however, a series of additional issues must be considered by both the translators and the voice-overs. As De Linde and kay (1999) believe, this is not a simply linguistic process. In fact they (ibid., p. 45) argue that these processes are "influenced by the material structure of a program and the semiotic relations operating between text and image, which must be processed by viewers." This is to support Gambier (1996), coining the term 'audio-visual language transfer' due to the interdependency of linguistic and visual information in order to describe any practice involving the transfer of linguistic content within an AV context.

There are many types of AV language transfer, which dubbing and subtitling are just two of the most relevant ones. In De Linde and Kay's (1996, p.46) definition, subtitling "supplements the dialogue of a film with written captions, while dubbing entirely substitutes an original dialogue with a phonetically-tuned synchronous oral translation." According to Gambier (1996, p. 9), these two common procedures of AV language transfer have some sub-categories, presented as follows:

- Simultaneous subtitling (for live interviews and news broadcasts, etc.)

- Synchronous dubbing

- Simultaneous interpretation, which can take three possible forms:

- live and often with summarizing as, for example, on the radio,

- $\quad$ pre-edited, much like a voice-over,

- $\quad$ in long distance two-way television interviews or at teleconferences.

Voice-over, where the translation tends to be 
reasonably faithful to the original and quasi synchronous.

- Narration, where a text read by a professional reader, actor or journalist is translated and sometimes condensed in advance and where the original dialogue is evanescent. Narration can be contrasted with voiceover, which attempts to be approximately simultaneous.

- Commentary, whereby a program is adapted to suit a new audience, with the possibility of information being added and omitted.

- Synchronization is achieved in relation to the images rather than the original dialogue, which is erased.

With all these sub-categories in an AV language transfer procedure, the question still remains as to what strategies translators mostly apply in order to render a script. These strategies are commonly divided into explicitation, implicitation, foreignization, domestication, etc., ones. A number of scholars and Translation Studies researchers have already worked on these concepts, presenting meta-models and frameworks on the related notions. Concerning the explicitation strategies, Klaudy's (2008) model seems to be a sufficient one, as it puts all the elements involved thoroughly into consideration. The present study then aimed at investigating these explicitation strategies through a frequency analysis with special reference to movie dubbing on the one hand, and movie subtitling on the other. In line, the present study aimed at finding a suitable answer to the following research question:

- Which of the explicitation strategies pointed out by Klaudy (2008) is the highest frequent, concerning the dubbed and the subtitled versions of the movie "Harry Potter and the Philosopher's Stone"?

\section{The Literature}

\subsection{On Explicitation}

Explicitation is defined by Vinay and Darbelnet (1995, p. 8) as "the process of introducing information into the target language which is present only implicitly in the source language, but which can be derived from the context or the situation." As such, the process could concern any kind of textual material whatsoever. What then became known as the 'explicitation hypothesis' was formulated by Blum-Kulka in 1986. In its historical development, the hypothesis broadly states that a translation will be more explicit than a corresponding non-translation, which may be either the source text or a parallel text in the target language. Blum-Kulka's (2001) original claim was based on cohesion markers:

The process of interpretations performed by the translator on the source text might lead to a TL [target language] text which is more redundant than the source text. This redundancy can be expressed by a rise in the level of cohesive explicitness in the TL text. This argument may be stated as the explicitation hypothesis, which postulates an observed cohesive explicitness from SL [source language] to TL texts regardless of the increase traceable to differences between the two linguistic and textual systems involved. It follows that explicitation is viewed here as inherent in the process of translation (p. 300).

It is conceivable that language pair-specific (i.e., non-universal) explicitations co-exist in translation with translation-inherent (i.e., universal) explicitations. This is the standpoint taken by Klaudy (2008), who assumes four different kinds of explicitation in translation. She (ibid.)has classified explicitation into 'obligatory' (due to syntactic and semantic differences between the SL and the TL), 'optional' (due to differences between text-building strategies and stylistic preferences of the two languages in question), 'pragmatic' (due to the need to bridge the cultural gap in translation) and 'translation-inherent' (resulting from the process of translation itself).

\subsection{Some Empirical Work on Dubbing and Subtitling in Iran}

Many of the studies done on subtitling and dubbing focused on translation strategies used in these processes and the frequency and adequacy of each strategy. These studies tried to classify different translation strategies and find linguistic dimensions affecting the quality of subtitling and dubbing. They also tried to suggest possible solutions to enhance the quality of subtitling and dubbing. These studies have rarely worked on explicitation in subtitling and dubbing directly. Some examples of such studies are presented in this section.

Regarding strategies used in translation of inter-lingual subtitling, Ghaemi and Benyamin (2010) tried to identify the inter-lingual subtitling strategies employed to translate English subtitles into Persian and determine their frequency. The researchers selected an English-Persian parallel corpus of 932 frames. The corpus comprised 100 minutes of film dialogues in English and 100 minutes of Persian subtitles. The corpus consisted of English audio scripts of five English feature films along with their Persian translations in the form of subtitles. The films were The Young Victoria directed in 2008 by Jean-Marc Vallee, August Rush directed in 2007 by Kirsten Sheridan, The Grudge 2 directed in 2006 by Takashi Shimizu, Big Fish directed in 2004 by Tim Burton, and $A$ Cinderella Story directed in 2004 by Mark Rosman. The selected films were among different genres. To analyze the data, the researchers used the classification of inter-lingual subtitling strategies proposed by Gottlieb (1992) as the theoretical framework. The strategies were expansion, paraphrase, transfer, imitation, transcription, dislocation, condensation, decimation, deletion and resignation. They selected frame - a word or a stretch of words in the form of subtitles which appear for a length of time on the screen and disappear before another frame appears - as the unit of analysis. They used frequency tables to report translation 
strategies used in each film and total number of translation strategies for all movies. The analysis of the result showed that different strategies including transfer $(46.06 \%)$, imitation (13.44\%), paraphrase 99.65\%), deletion (8.54\%), expansion (6.03\%), condensation (3.44\%), dislocation $(2.41 \%)$, resignation $(1.85 \%)$, transcription $(0.81 \%)$, and decimation $(0.81 \%)$ were used in transferring the expressions of the original films. The findings of the study showed that all Gottlieb's (1992) proposed strategies were used in English-Persian subtitling of English feature films with some degree of variation. They concluded that the film genre played a crucial role in the variation of the used strategy. They also concluded that the subtitles transferred as much information as possible by translating the dialogues completely and accurately.

VahidDastjerdi and Rahekhoda (2010) tried to classify different types of expansions used in subtitling and investigate the appropriateness and inappropriateness of the application of each type considering the time and space constraints which are peculiar to subtitling. In line with the goal of the study, the researchers selected three English films - The Net (1995), Contact (1997), and Mission Impossible 2 (2000) - available with Persian subtitles. The rationale behind their selection was that the quality of the Persian subtitles was excellent. The researchers used the following procedures to answer the research question. First, they watched the selected films, identified and extracted subtitles in which expansion had been employed. Then, they categorized Persian subtitles based on the reason that caused expansion. Next, they investigated appropriateness and inappropriateness of using each type of expansion in subtitles. After that, they proposed a shorter equivalent not containing expansion for most of the original English dialogues to show that the meaning can be fully conveyed in most cases without resorting to expansion. Finally, they compared subtitles and proposed translations in terms of lexical density by calculating the number of characters. Having analyzed the data, the researchers identified the following types of expansion: expansion caused by explicitation of co-textual information (29.45\%), expansion caused by paraphrasing $(25.4 \%)$, expansion caused by subtitler's preference (19.6\%), expansion caused by explicitations of visual information (13.75\%), expansion caused by mistranslation (5.9\%), and expansion caused by explicitation of contextual information (5.9\%). According to Seguinot (1988, as cited in Klaudy, 2008), explicitation is additions in a translated text which cannot be explained by structural, stylistic, or rhetorical differences between two languages. The result of the study showed that explicitation, mistranslation, and paraphrasing gave rise to the expansion of subtitles in the films under investigation. The result also showed that the application of expansion was not justified and appropriate in most cases since correct and shorter equivalents which were equally capable of conveying the intended meaning could be posited for the original dialogues. The researchers concluded that subtitles are just one channel of information in a poly-semiotic text like a film which includes other semiotic channels such as pictures, music, and sound effect. Thus, subtitlers should not include everything in the subtitles, and should let viewers receive part of the information from other channels.

\subsection{Global Empirical Work on Dubbing and Subtitling}

Subtitling is often assumed to be a solely linguistic operation which involves the translation of dialogue into written captions. However, from a viewer's perspective subtitled productions include more than just linguistic information. In addition to subtitles, viewers have to process film images in order to establish a coherent narrative. Both types of information must be received through the same visual channel. In this regard, De LindeKay (1999) examined a number of significant linguistic and non-linguistic features of subtitles and film which potentially affect the way viewers watch subtitled productions. Non-linguistic features were examined in the context of recent studies on eye-movement behavior, including a comparative study involving deaf and hearing viewers conducted by the authors.

In a study conducted by Pelsmaekers and Van Besien (2002), the researchers set out to examine empirically how humorous ironyfares in the constrained form of translation that subtitling was, and whether these observations could be related to known humortranslation and subtitling strategies. Drawing on a speech act approach to irony the researchers studied 211 humorous ironic utterances and their Dutch subtitles from twelve episodes of the 'Blackadder'series. While a quantitative approach showed that nearly all these subtitles retained some degree of ironic potential, it was also found that in about two thirds of these cases verbal ironic cues were influenced by the translation in interesting ways. A qualitative analysis showed that on the whole, the Dutch subtitles were often more explicitly critical. This means that from the known strategies of irony translation only a few turned out to be very important in these data. Part of this could be explained by subtitling strategies reported in the literature, which generally seemed to work against the preservation of a particular kind of ironic cues, i.e. those that were found to have an interpersonal rather than referential function.

The authenticity of fictional dialogue is widely held to play a pivotal role in shaping the audience's perception of the quality of a film. Yet the factors that account for the authenticity of both original and dubbed film conversations remain largely under-researched. In this regard, Perez-Gonzalez'es (2007) paper began by outlining key contributions from the fields of stylistics, film studies and corpus-based translation studies that have enhanced our understanding of the specific nature and dynamics of fictional dialogue and its translation. A common assumption that underpins these approaches is that the success ofthe narrative and characterization-enhancing resources deployed in a film is contingent on the build-up of interpersonal alignments through a combination of prefabricated orality and spontaneous-sounding conversation. And yet both film 
theory and dubbing studies have so far focused on phenomena that take place within a single turn-at-talk and hence neglected the study of the sequential dimension of film dialogue. Drawing on the analysis of four scenes of the English and Spanish versions of Twelve Angry Men, this article attempted to demonstrate the advantages of Martin's (2000) systemic functional modeling of the exchange, especially his notion of 'telos'. Ultimately, this paper assessed the advantages of a heightened awareness of the sequential configuration of dialogue among dubbing practitioners.

\section{Method}

\subsection{Materials}

The movie "Harry Potter and the Philosopher's Stone" along with its corresponding Persian versions in both dubbed and subtitled versions were chosen as the materials of the study. It is the first novel in the Harry Potter series written by J. K. Rowling (1997), featuring Harry Potter, a young wizard. It describes how Harry discovers that he is a wizard, makes close friends and a few enemies at the Hogwarts School of Witchcraft and Wizardry, and with the help of his friends thwarts an attempted comeback by the evil wizard Lord Voldemort, who killed Harry's parents when Harry was one year old.One of the reasons for such selection was that both versions of its Persian translation, i.e., the dubbed and the subtitled versions were available on the market. The other reason relates to the length of the movie, which could provide grounds for the model under investigation to be tested.

\subsection{Procedure}

After watching the movie, the Source Text (ST) was compared with the Persian dubbed version. Then, the same procedure was carried out for comparing the ST to Persian subtitled version. For the analysis of the aforementioned corpora, Klaudy's (2008) classification of explicitation was employed. As mentioned before, this classification introduces four strategies. These include:

1) Obligatory explicitations (S1)are caused by grammatical differences between source and target languages. They occur when the translator is forced by these differences to spell things out explicitly that are only implicit in the source text.

2) Optional explicitations (S2) are the result of "differences in text-building strategies [...] and stylistic preferences between languages. Such explicitations are optional in the sense that grammatically correct sentences can be constructed without their application in the target language, although the text as a whole will be clumsy..." (Klaudy, 2008, p. 106).

3) Pragmatic explicitations (S3)are due to differences in cultural and/or world knowledge that members of source and target language communities share. They typically occur when a translator needs to add linguistic material to explain a concept specific to the source culture.

4) Translation-inherent explicitation (S4)“can be attributed to the nature of the translation process itself" (Klaudy, 2008, p. 107).

Having found the cases of explicitation in each Target Text (TT) version, the frequencies of explicitation items in the dubbed version were compared to those of the subtitled one in order to find out which version was more explicitly rendered. In addition, to figure out what explicitation strategy held the highest frequency of usage was another purpose of the study.

\section{The Data}

To begin with, the dubbed and subtitled versions of the movie were carefully watched. Then, each of the explicitations occurred within the process of translation were highlighted and further placed in four different explicitation strategies under investigation. Examples of strategies detected relating to the dubbed and the subtitled versions are presented in Appendices A and B respectively. The related data are presented from a descriptive perspective as shown in Table 1..

Table 1. A General Overview on the Frequencies of the Explicitation Strategies Pointed Out by Klaudy (2008) in the Movie "Harry Potter and the Philosopher's Stone"

\begin{tabular}{ccc}
\hline Strategy Type & $\begin{array}{c}\text { Frequency in Dubbed } \\
\text { Version }\end{array}$ & $\begin{array}{c}\text { Frequency in Subtitled } \\
\text { Version }\end{array}$ \\
\hline S1 & 27 & 53 \\
S2 & 4 & 55 \\
S3 & 2 & 11 \\
S4 & 4 & 14 \\
Total & 37 & 133 \\
\hline
\end{tabular}

Having a general overview of the collected data, Table 1.presents interesting results, regarding the frequency of each explicitation strategy usage. As suggested in Table 1., the four introduced strategies pointed out by Klaudy (2008) mostly held higher frequencies of usage in subtitled version of the movie. This was in contrast with the frequencies of the strategies applied in dubbing the movies. Figure 1.illustrates the data gathered from a descriptive perspective: 


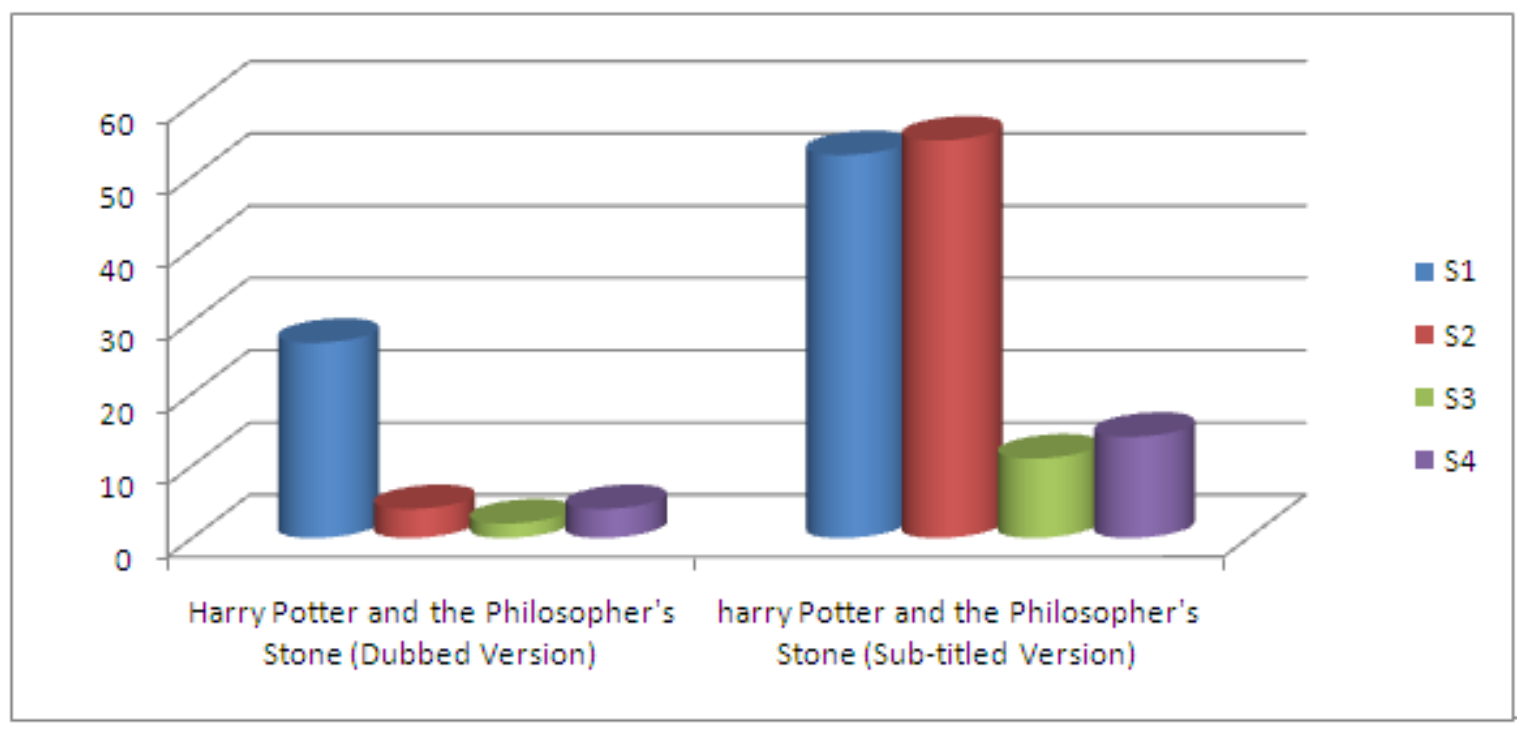

Figure 1. A Descriptive Overview on the Collected Data

To see whether these extents were of any statistical significance, the Chi-square procedure was used. Table 2.presents the basic statistical information about the frequencies of explicitation strategies for this movie.

Table 2. The Basic Descriptive Information on the Frequencies of the Explicitation Strategies

\begin{tabular}{cccc}
\hline & Observed N & Expected N & Residual \\
\hline 1 & 37 & 85.0 & -48.0 \\
2 & 133 & 85.0 & 48.0 \\
Total & 170 & & \\
\hline
\end{tabular}

Following the basic information on the frequencies based on Klaudy's (2008) explicitation strategies, Table 3. presents the results of the Chi-square test run to see if the differences were statistically significant.

Table 3. Chi-Square Test Results for the Differences Among the Frequencies of Explicitation Strategies

\begin{tabular}{cc}
\hline Chi-Square & $54.212^{\mathrm{a}}$ \\
\hline $\mathrm{df}$ & 1 \\
Asymp. Sig. & .000 \\
\hline
\end{tabular}

The results of the Chi-square test revealed statistically significant differences among the frequencies of the strategies applied. In other words, there were statistically significant differences among the frequencies of explicitation strategies comparing the dubbed to the subtitled version. Based on the obtained results, it would be interesting to point out that S1 (obligatory explicitation) held the highest frequency of use as compared to the other three strategies introduced by Klaudy (2008).

\section{Discussion and Conclusion}

Concerning the afore-posed research question, the results of the study revealed that S1 held the highest frequencies among the other three types introduced. In addition, this was true for both versions of the movies, i.e., the dubbed and the subtitled versions. Obligatory explicitations are mostly referred to as linguistic-bound ones (Klaudy, 2008). In other words, linguistics barriers related to the nature and the family of languages (Yule, 1996) could possibly have an impact on the frequency of a translation strategy.

The results of the present work supported those of De Linde and Kay's (1999) work on dubbing and subtitling, in which, the researchers examined a number of significant linguistic and non-linguistic features of subtitles and film which potentially affected the way viewers watch subtitled productions. Non-linguistic features were then examined in the contextof recent studies on eye-movement behavior, including a comparative study involving deaf and hearing viewers conducted by the authors.

The basic foundation of the study supported Pelsmakers and Van Besien's (2002) work, in which, an approach to irony was made. In this regard, the researchers studied 211 humorous ironic utterances and their Dutch subtitles from twelve episodes of the 'Blackadder'series ('Blackadder II' and 'Blackadder the Third'). While a quantitative approach showed that nearly all these subtitles retained some degree of ironic potential, the researchers also found that in about two thirds of these cases, verbal ironic cues were influenced by the translation in interesting ways. In addition, a qualitative analysis showed that on the whole, the Dutch subtitles were often more explicitly critical. This means that from the known strategies of irony translation, only a few turned out to be very important in these data. Part of this could be explained by subtitling strategies reported in the literature of the study, which generally seemed to work against the preservation of a particular kind of ironic cues, i.e. those that were found to have an interpersonal rather than referential function. 
The methodology used in this paper was similar to the one applied by Jafari (2013), in which, the author investigated the approaches and strategies employed by Iranian subtitlers in the translation of metaphors into Persian. The material gathered for this purpose consisted of six subtitled films. The films were selected among those originally produced in English and subtitled to Persian. In order to have a contrastive analysis of metaphors and their translations, the movies dialogues and their subtitles were contrasted. At the first stage, metaphor was defined and with the definition in mind, metaphors in the movies were identified. After determining metaphors in each film, the translations of the metaphors needed to be identified and matched with the original ones. To this aim, the original metaphors and their Persian subtitled translations were compared and contrasted in order to track down the translations of the identified metaphors. Afterwards, the metaphors and their translations were listed in tables to investigate the most and the least frequent approaches and strategies employed by Iranian subtitlers in dealing with metaphors.

As the results of the present work revealed, obligatory explicitation was at the highest frequency of use, compared with the other three types of strategies. This might partly be due to the nature of languages (Yule 1996, Brown 1980). In klaudy's definition (2008, p. 106), these strategies are "caused by grammatical differences between source and target languages. They occur when a translator is forced by these differences to spell things out explicitly, while they are only implicit in the source text." Thus, it might be concluded that this type of strategy was the most frequent one due to the grammatical differences among English and Persian.

The findings of the present work have the potentialities of acting as a tool to understanding the phenomena of dubbing and subtitling issues better. As Venuti (2004) pointed out, from 1990s onward, an increasing interest has been paid to the process- oriented research programs. Accordingly, translation strategies play a significant role in this regard. Thus, it is hoped that the present thesis project would be able to informing work provided on the issue.

\section{REFERENCES}

[1] Blum-Kulka, S. (2001).Shifts of cohesion and coherence in translation. First published in 1986 in Interlingual and Intercultural Communication. Discourseand cognition in translation and second language acquisition, eds. Juliane House and Shoshana Blum-Kulka.Tübingen: Narr. 17-35. Cited here from The Translation Studies Reader, ed. Lawrence Venuti. London and New York:Routledge. 298-313.

[2] Brown. H. D. (1980). Principles of language learning and teaching. London: Prentice Hall Inc.

[3] De Linde, Z., \& Kay, N. (1999). Processing subtitles and film images: Hearing vs. deaf viewers. The Translator 5 (1), pp. 45-60.

[4] Ghaemi, F., \& Benyamin, J. (2010). Strategies used in the translation of inter-lingual subtitling. Journal of English Studies, pp.39-49. Retrieved June 22, 2012, from http://www.sid.ir/en/VEWSSID/J_pdf/1024220100103.pdf

[5] Jafari, O. (2013). Strategies of translating metaphors in foreign movies employed by Iranian subtitlers. Retrieved November 2013 from http://www.translationdirectory.com/articles/article2439.php

[6] Klaudy, K. (2008): Explicitation.Mona Baker, Gabriela Saldanha (Eds). (pp. 106-107)Routledge encyclopedia of translation studies. London: Routledge.

[7] Pelsmaker, K., \& Van Besien, F. (2002).Subtitling irony: Blackadder in Dutch. The Translator, 8 (2), pp. 241-266.

[8] Perez-Gonzalez, L. (2007). Appraising dubbed conversation: Systemic functional insights into the construal of naturalness in translated film dialogue. The Translator, 13 (1), pp. 1-38.

[9] Venuti, L. (2004). The translation studies reader. New York: Routledge.

[10] Vinay, J., \&Darbelnet, J. (1995). A comparative stylistics of French and English: A methodology for translation. In J. Munday, Introducing translation studies: Theories and application. London and New York: Routledge.

[11] VahidDastjerdi, H., \&Rahekhoda, R. (2010). Expansion in subtitling: The case of three English films with Persian subtitles. Journal of Language and Translation, pp. 2-27. Retrieved June 23, 2012, from http://www.unish.org/upload/word/2010v11-01.pdf

[12] Yule, G. (1996). The study of language (2nd ed.). Cambridge: Cambridge University Press. 